\title{
Line x Tester Analysis for Heterosis and Inbreeding Depression in Bottle Gourd (Lagenaria siceraria (Mol) Standl.)
}

\author{
Sandeep K. Mauriya* and A. K. Pal \\ Department of Horticulture, Institute of Agricultural Sciences, Banaras Hindu University, \\ Varanasi (UP), India \\ *Corresponding author
}

A B S T R A C T

Keywords

Heterosis, Bottle gourd, Inbreeding depression, Additive gene action

\section{Article Info}

Accepted:

07 January 2021

Available Online:

10 February 2021
In the present investigation, 10 lines and 4 testers of bottle gourd were taken in line $\mathrm{x}$ tester analysis for analyzing magnitude of heterosis and degree of inbreeding depression observed for 10 characters of bottle gourd. Some crosses showed high degree of heterosis coupled with high degree of inbreeding depression indicating thereby that there is a greater role non-additive gene action controlling the expression of those characters. In contrast, there are few crosses where low heterosis was linked with high degree of inbreeding depression. This indicates that probably gene having additive effects are cancelled, leading to appreciable inbreeding depression for such characters. In addition, crosses showing high degree of heterosis are linked with low degree of inbreeding depression indicating that, possibly, gene with additive as well as non additive gene action play important role in expression of heterosis in such crosses.

\section{Introduction}

Bottle gourd [Lagenaria siceraria (Mol.) Standl] is one of the important cucurbits commonly grown in both rainy and summer season in various parts of India. The chromosome number of bottle gourd is $2 \mathrm{n}=$ $22(X=11)$.The fresh fruit has light green smooth skin and white flesh. They come in a variety of shapes. They can be huge and round, small and bottle shaped or slim and serpentine, some times more than a meter long. It is a monoceious annual having vine with a long ribbed stem and strong tendrils. It is also called white flower gourd as it produces large white male flowers on a long peduncle. Andromonoecious lines were also reported in bottle gourd (Hari Har Ram, 1999).

Bottle gourd is one of the best vegetables and is rich in medicinal value. It cures many disorders of our body which occurs because of our bad eating habits. Bottle gourd is the highly nutritive for human diet because it is a valuable source of carbohydrates, proteins, vitamins and minerals. $100 \mathrm{~g}$ edible bottle gourd fruit contains $2.9 \%$ carbohydrates, 
$0.25 \%$ proteins, $0.5 \%$ fats and $0.5 \%$ minerals such as potassium, calcium, phosphorous and iron.

Heterosis could be a composite/complex genetical phenomenon showed within the predominance of $F_{1}$ hybrids over its parents in terms of yield (or) some other traits. Sometimes, hybrid vigour is used as a synonym for heterosis. It is widely accepted that hybrid vigour represents only hybrid's superiority over parents, whereas heterosis also represents certain other conditions. However, the dominance of the hybrids upon parents is the maximum cases of heterosis. It may be expressed in term of mid parent (MP) or over the better parent (BP) or over the standard check (SC) (Hayes et al., 1955) with respect to agronomically useful traits. The primary objective of heterosis breeding is to achieve a quantum jump in yield and quality traits of crop. The high degree of heterosis is shown by the hybrids over better parents as well as over commercial variety (Thakur et al., 2017). Heterosis is a natural phenomenon whereby hybrid offspring from geneticallydiverse individuals show increased vigour relative to their parents (Shull, 1948).

\section{Materials and Methods}

The experiment was conducted at Horticulture Research Farm of the Department of Horticulture, Institute of Agricultural Sciences, Banaras Hindu University, Varanasi, during rainy-summer and rainy season of 2017 and 2018. The experimental material of this investigation comprising 54 genotypes. Out of 14 genotypes, 10 geneotypes were used as lines and 4 as testers under Line $\times$ Tester mating design producing 40 crosses for study of heterosis and inbreeding depression. (Kempthorne, 1957) for 10 characters of bottle gourd. seeds were sown at $2 \times 0.75 \mathrm{~m}$ spacing in Randomized Block Design with three replications. The selected 10 parental lines i.e. $\mathrm{L}_{1}$ (Pant BG-1),
$\mathrm{L}_{2}$ (Pusa Samaridhi), $\mathrm{L}_{3}$ (Narendra Pratibha), $\mathrm{L}_{4}$ (KBGL-1), L5 (KBGL-19), L 6 (KBGL-21), $\mathrm{L}_{7}$ (KBGL-22), L 8 (Kalyanpur Long Green (KLG), $\mathrm{L}_{9}$ (Azad Harit) and $\mathrm{L}_{10}$ (Kalyanpur Long Green (KLG) and 4 testers i.e., $\mathrm{T}_{1}$ (Pusa Samaridhi), $\mathrm{T}_{2}$ (Narendra Reshami), $\mathrm{T}_{3}$ (Kashi Ganga) and $\mathrm{T}_{4}$ (Arka Bahar) along with one check (Pusa Naveen) were used. All the recommended agronomic package of practices and protection measures were followed to raise a good crop. Fertilizers and manures were applied as per recommended dose.

Observations on metric traits were recorded on single plant basis on five random selected competitive plants in each genotype from each plot for all the traits separately for 10 quantitative characters viz. Days to $1^{\text {st }}$ male flower opening, days to $1^{\text {st }}$ female flower opening, days to $50 \%$ male flowers, days to $50 \%$ female flowers, days to first fruit harvest, fruit length $(\mathrm{cm})$, fruit diameter $(\mathrm{cm})$, average fruit weight $(\mathrm{kg})$, fruit flesh thickness $(\mathrm{cm})$ and rind thickness $(\mathrm{mm})$.

\section{Results and Discussion}

Out of 40 crosses derived from crosses made between 10 lines and 4 testers, heterosis has been estimated over mid, better and economic parents for all the 10 characters studied. Simultaneously, extended inbreeding depression has been studied for the same traits. It is obvious from (Table 1) the extent of heterosis for days to $1^{\text {st }}$ male flower over mid, better and economic parent are depicted from this study. The mid parent heterosisee for this characters is significant in three crosses like KBGL-19 x Pusa Samaridhi, Narendra Pratibha x Narendra Rashmi, Kalyanpur Long Green x Kashi Ganga. Out of these three, only one cross-Narendra Pratibha $\mathrm{x}$ Narendra Rashmi showed significant negative heterosis, which is desirable for this character. 
Table.1 Extent of heterosis and inbreeding depression for days to $1^{\text {st }}$ male flower, days to $1^{\text {st }}$ male flower days to $50 \%$ male flowers

\begin{tabular}{|c|c|c|c|c|c|c|c|c|c|c|c|c|c|}
\hline \multirow[t]{2}{*}{ S.N. } & \multirow[t]{2}{*}{ Crosses } & \multicolumn{4}{|c|}{ Days to 1st male flower opening } & \multicolumn{4}{|c|}{ Days to first female flowers opening } & \multicolumn{4}{|c|}{ Days to $50 \%$ male flowers } \\
\hline & & MPH & $\mathrm{HB}$ & $\mathrm{EH}$ & ID & MPH & $\mathrm{HB}$ & EH & ID & MPH & $\mathrm{HB}$ & EH & ID \\
\hline 1. & L1 x T1 & -0.28 & - & - & -1.55 & -0.64 & - & - & -4.63 & -0.34 & - & - & 0.34 \\
\hline 2. & L2 $\times$ T1 & -2.12 & -0.57 & - & -1.88 & -2.58 & - & - & 0.14 & -0.35 & - & - & -1.75 \\
\hline 3. & L3 x T1 & -5.11 & -4.64 & -0.15 & $-7.52 *$ & -0.43 & - & - & -0.13 & $-3.66^{*}$ & -2.66 & - & $-5.46^{*}$ \\
\hline 4. & L4 x T1 & 2.96 & - & - & -2.19 & -3.08 & -3.05 & - & $-6.43^{*}$ & $-3.90^{*}$ & -3.85 & - & -1.88 \\
\hline 5. & L5 x T1 & $5.96^{*}$ & - & - & -0.53 & 2.24 & - & - & -0.52 & -1.74 & -1.02 & - & 0.11 \\
\hline 6. & L6 x T1 & 2.79 & - & - & -0.27 & 0.36 & - & - & 0.92 & $-5.44 * *$ & $-5.33^{*}$ & 0.00 & $-4.92 *$ \\
\hline 7. & L7 x T1 & -4.83 & -4.04 & - & $-7.69^{*}$ & 0.03 & - & - & 0.53 & -2.70 & -1.17 & - & -1.42 \\
\hline 8. & L8 x T1 & 1.34 & - & - & -3.48 & -2.64 & -0.86 & - & -2.94 & -1.13 & -1.02 & - & -1.95 \\
\hline 9. & L9 x T1 & 4.64 & - & - & -1.68 & 0.83 & - & - & -1.98 & -3.43 & -2.60 & - & -0.12 \\
\hline 10. & L10 x T1 & 0.42 & - & - & -2.08 & -0.96 & -0.93 & - & -3.35 & -1.15 & - & - & -1.16 \\
\hline 11. & L1 x T2 & -0.84 & - & - & -2.40 & -1.83 & - & - & -0.41 & 1.14 & - & - & -1.69 \\
\hline 12. & L2 $\times$ T2 & -3.67 & -1.87 & - & 0.29 & -1.45 & - & - & -1.12 & 0.35 & - & - & $-5.70 *$ \\
\hline 13. & L3 x T2 & $-5.37 *$ & -4.64 & -0.15 & -0.81 & -4.95 & -4.44 & - & -0.90 & 0.11 & - & - & $-5.10 *$ \\
\hline 14. & $\mathrm{~L} 4 \times \mathrm{T} 2$ & 3.94 & - & - & -2.30 & 0.66 & - & - & -1.19 & $-3.69^{*}$ & -3.31 & - & -1.89 \\
\hline 15. & L5 x T2 & 2.45 & - & - & -1.50 & 5.01 & - & - & 1.21 & $-4.00^{*}$ & -2.96 & - & -3.06 \\
\hline 16. & L6 x T2 & -2.78 & -2.51 & - & $-8.43^{* *}$ & -3.70 & -3.32 & - & -2.19 & $-3.75^{*}$ & -3.53 & - & -4.73 \\
\hline 17. & L7 x T2 & -0.96 & -0.42 & - & -2.09 & -0.07 & - & - & -1.32 & -0.82 & - & - & -2.05 \\
\hline 18. & L8 $\times$ T2 & -5.00 & -3.43 & -0.59 & $-9.33^{* *}$ & -5.08 & -3.32 & - & $-7.54^{*}$ & 1.48 & - & - & 1.46 \\
\hline 19. & L9 x T2 & -1.05 & - & - & -5.32 & $-5.91 *$ & -5.73 & - & $-7.91 *$ & $-4.56^{*}$ & -3.42 & - & -1.59 \\
\hline 20. & $\mathrm{~L} 10 \times \mathrm{T} 2$ & 3.62 & - & - & 2.55 & 2.12 & - & - & -0.78 & -0.46 & - & - & -2.08 \\
\hline 21. & L1 x T3 & 4.87 & - & - & 0.67 & 2.38 & - & - & -2.65 & -3.35 & -2.85 & - & -4.69 \\
\hline 22. & L2 x T3 & 2.21 & - & - & -3.20 & 0.90 & - & - & -5.21 & 0.29 & - & - & $-6.49 * *$ \\
\hline 23. & L3 x T3 & 1.13 & - & - & -4.74 & -0.79 & - & - & -2.67 & -2.80 & -1.62 & - & $-7.52 * *$ \\
\hline 24. & L4 x T3 & 5.18 & - & - & -1.62 & 1.40 & - & - & 1.12 & $-4.18^{*}$ & $-4.07 *$ & - & -0.94 \\
\hline 25. & L5 x T3 & 3.10 & - & - & -1.64 & -1.07 & -0.81 & - & $-7.59 *$ & $-6.73^{* *}$ & $-6.21 * *$ & -0.36 & $-8.18 * *$ \\
\hline 26. & L6 x T3 & 3.15 & - & - & -4.28 & 2.92 & - & - & 0.90 & -1.30 & -1.02 & - & -0.57 \\
\hline 27. & L7 x T3 & -0.07 & - & - & -4.52 & -3.26 & -2.67 & - & $-9.89 * *$ & $-4.02 *$ & -2.34 & - & -3.95 \\
\hline 28. & L8 x T3 & $5.40 *$ & - & - & -0.67 & -2.61 & -0.40 & - & -5.50 & $-5.15^{* *}$ & $-4.88 *$ & - & -2.15 \\
\hline 29. & L9 x T3 & 3.54 & - & - & 0.68 & 0.20 & - & - & 2.33 & $-5.49 * *$ & $-4.85^{*}$ & - & $-5.28 *$ \\
\hline 30. & $\mathrm{~L} 10 \times \mathrm{T} 3$ & 1.19 & - & - & -0.14 & 0.26 & - & - & -1.53 & -2.35 & -0.93 & - & $-6.21 *$ \\
\hline 31. & $\mathrm{~L} 1 \times \mathrm{T} 4$ & -0.35 & - & - & -1.82 & -0.81 & - & - & -1.76 & -2.14 & -0.93 & - & -3.89 \\
\hline 32. & L2 x T4 & 3.17 & - & - & -1.78 & 1.92 & - & - & $-8.99 * *$ & -0.18 & - & - & $-5.33^{*}$ \\
\hline 33. & L3 x T4 & 0.56 & - & - & -3.33 & -3.61 & -3.54 & - & -2.99 & -1.92 & -1.40 & - & -1.54 \\
\hline 34. & $\mathrm{~L} 4 \times \mathrm{T} 4$ & 3.02 & - & - & 0.07 & -0.99 & -0.40 & - & -1.33 & -2.18 & -0.58 & - & -0.88 \\
\hline 35. & L5 x T4 & 3.63 & - & - & -0.40 & 2.85 & - & - & 1.16 & -2.28 & -0.00 & - & -1.29 \\
\hline 36. & L6 $\times$ T4 & 1.87 & - & - & -0.27 & -1.38 & -1.18 & - & -4.53 & -3.51 & -2.10 & - & $-6.56^{* * *}$ \\
\hline 37. & L7 x T4 & -0.76 & -0.28 & - & -0.28 & -4.74 & -4.36 & - & -4.14 & -3.39 & -3.39 & -0.84 & -3.63 \\
\hline 38. & L8 $\times$ T4 & 3.23 & - & - & -3.95 & -3.30 & -2.10 & - & -2.68 & $-4.78^{* *}$ & -3.39 & -0.84 & $-5.80^{*}$ \\
\hline 39. & L9 x T4 & -3.09 & -1.71 & - & $-7.96^{*}$ & -3.30 & -2.53 & - & -0.20 & $-5.59 * *$ & -3.27 & -0.72 & -4.11 \\
\hline 40. & L10 x T4 & -1.60 & -1.12 & - & -1.34 & 0.07 & - & - & -1.58 & 2.97 & - & - & 1.13 \\
\hline
\end{tabular}


Table.2 Extent of heterosis and inbreeding depression for days to 50\% female flowers, days to first fruit harvest and Fruit length (cm)

\begin{tabular}{|c|c|c|c|c|c|c|c|c|c|c|c|c|c|}
\hline \multirow[t]{2}{*}{ S.N. } & \multirow[t]{2}{*}{ Crosses } & \multicolumn{4}{|c|}{ Days to $50 \%$ female flowers } & \multicolumn{4}{|c|}{ Days to first fruit harvest } & \multicolumn{4}{|c|}{ Fruit length (cm) } \\
\hline & & MPH & $\mathrm{HB}$ & EH & 0.34 & $\mathrm{HB}$ & EH & ID & ID & HPH & HB & $\mathrm{EH}$ & ID \\
\hline 1. & L1 x T1 & -0.34 & - & - & -1.75 & - & - & 0.34 & $-4.99^{*}$ & -0.18 & - & 0.54 & 2.69 \\
\hline 2. & L2 $\times$ T1 & -0.35 & - & - & $-5.46^{*}$ & - & - & -1.75 & 0.74 & 7.79 & 5.81 & $12.88 *$ & 0.27 \\
\hline 3. & L3 x T1 & $-3.66^{*}$ & -2.66 & - & -1.88 & -2.66 & - & $-5.46^{*}$ & -1.22 & -2.74 & - & 1.56 & -1.30 \\
\hline 4. & L4 x T1 & $-3.90^{*}$ & -3.85 & - & 0.11 & -3.85 & - & -1.88 & -1.51 & 3.27 & 1.64 & 4.45 & 4.21 \\
\hline 5. & L5 x T1 & -1.74 & -1.02 & - & $-4.92 *$ & -1.02 & - & 0.11 & -2.24 & -1.06 & - & 1.44 & -0.71 \\
\hline 6. & L6 x T1 & $-5.44 * *$ & $-5.33^{*}$ & 0.00 & -1.42 & $-5.33^{*}$ & 0.00 & $-4.92 *$ & -2.15 & $-8.66^{*}$ & - & - & 0.45 \\
\hline 7. & L7 x T1 & -2.70 & -1.17 & - & -1.95 & -1.17 & - & -1.42 & -0.12 & -1.23 & - & - & -1.52 \\
\hline 8. & L8 $\times$ T1 & -1.13 & -1.02 & - & -0.12 & -1.02 & - & -1.95 & -0.94 & 1.82 & - & - & 5.51 \\
\hline 9. & L9 x T1 & -3.43 & -2.60 & - & -1.16 & -2.60 & - & -0.12 & -0.47 & 1.77 & - & 0.60 & -2.03 \\
\hline 10. & L10 x T1 & -1.15 & - & - & -1.69 & - & - & -1.16 & -2.48 & $13.57^{* * *}$ & 3.86 & 6.74 & 7.10 \\
\hline 11. & L1 x T2 & 1.14 & - & - & $-5.70^{*}$ & - & - & -1.69 & 2.05 & 1.94 & 0.06 & 2.53 & 0.94 \\
\hline 12. & L2 $\times$ T2 & 0.35 & - & - & $-5.10^{*}$ & - & - & $-5.70^{*}$ & 0.93 & 2.42 & 0.39 & 7.10 & 3.60 \\
\hline 13. & L3 x T2 & 0.11 & - & - & -1.89 & - & - & $-5.10^{*}$ & -1.46 & -1.44 & - & 2.77 & 0.47 \\
\hline 14. & L4 x T2 & $-3.69^{*}$ & -3.31 & - & -3.06 & -3.31 & - & -1.89 & 0.23 & -4.56 & - & - & -6.37 \\
\hline 15. & L5 x T2 & $-4.00^{*}$ & -2.96 & - & -4.73 & -2.96 & - & -3.06 & 1.27 & -2.32 & - & 0.00 & -2.95 \\
\hline 16. & L6 x T2 & $-3.75^{*}$ & -3.53 & - & -2.05 & -3.53 & - & -4.73 & -1.31 & -1.87 & - & 0.76 & $11.50^{*}$ \\
\hline 17. & L7 x T2 & -0.82 & - & - & 1.46 & - & - & -2.05 & -4.45 & -0.12 & - & - & $12.35^{*}$ \\
\hline 18. & L8 $\times$ T2 & 1.48 & - & - & -1.59 & - & - & 1.46 & -0.30 & 8.74 & 0.06 & 2.53 & -4.81 \\
\hline 19. & L9 x T2 & $-4.56^{*}$ & -3.42 & - & -2.08 & -3.42 & - & -1.59 & -0.45 & $-9.60^{*}$ & - & - & -3.57 \\
\hline 20. & $\mathrm{~L} 10 \times \mathrm{T} 2$ & -0.46 & - & - & -4.69 & - & - & -2.08 & -1.72 & 3.49 & - & - & 0.37 \\
\hline 21. & L1 x T3 & -3.35 & -2.85 & - & $-6.49 * *$ & -2.85 & - & -4.69 & 0.12 & 0.99 & - & 4.15 & 1.39 \\
\hline 22. & L2 $x$ T3 & 0.29 & - & - & $-7.52^{* * *}$ & - & - & $-6.49 * *$ & -0.48 & -6.88 & - & - & 4.83 \\
\hline 23. & L3 $\times$ T3 & -2.80 & -1.62 & - & -0.94 & -1.62 & - & $-7.52 * *$ & -1.30 & -4.93 & - & 1.56 & -1.48 \\
\hline 24. & L4 x T3 & $-4.18^{*}$ & $-4.07 *$ & - & $-8.18^{* *}$ & $-4.07 *$ & - & -0.94 & -0.23 & 0.35 & - & 3.91 & 10.13 \\
\hline 25. & L5 $x$ T3 & $-6.73 * *$ & $-6.21 * *$ & -0.36 & -0.57 & $-6.21^{* *}$ & -0.36 & $-8.18 * *$ & -0.46 & -8.03 & - & - & 8.48 \\
\hline 26. & L6 x T3 & -1.30 & -1.02 & - & -3.95 & -1.02 & - & -0.57 & -1.16 & $-16.87 * *$ & - & - & $-15.13^{*}$ \\
\hline 27. & L7 $x$ T3 & $-4.02 *$ & -2.34 & - & -2.15 & -2.34 & - & -3.95 & -1.71 & 0.21 & - & 2.77 & 8.78 \\
\hline 28. & L8 $\times$ T3 & $-5.15^{* *}$ & $-4.88^{*}$ & - & $-5.28^{*}$ & $-4.88^{*}$ & - & -2.15 & $-6.09^{*}$ & 2.02 & - & - & 6.09 \\
\hline 29. & L9 x T3 & $-5.49 * *$ & $-4.85^{*}$ & - & $-6.21 *$ & $-4.85^{*}$ & - & $-5.28^{*}$ & -0.68 & -3.57 & - & - & 6.47 \\
\hline 30. & L10 x T3 & -2.35 & -0.93 & - & -3.89 & -0.93 & - & $-6.21^{*}$ & -1.05 & 3.25 & - & - & $11.73^{*}$ \\
\hline 31. & $\mathrm{~L} 1 \times \mathrm{T} 4$ & -2.14 & -0.93 & - & $-5.33^{*}$ & -0.93 & - & -3.89 & -3.09 & 1.99 & 0.12 & - & 0.24 \\
\hline 32. & L2 $\times$ T4 & -0.18 & - & - & -1.54 & - & - & $-5.33^{*}$ & -1.77 & 1.10 & - & 1.99 & -2.48 \\
\hline 33. & L3 $\times$ T4 & -1.92 & -1.40 & - & -0.88 & -1.40 & - & -1.54 & -0.68 & 2.24 & - & 2.83 & $13.46^{*}$ \\
\hline 34. & L4 x T4 & -2.18 & -0.58 & - & -1.29 & -0.58 & - & -0.88 & 0.34 & 7.54 & 5.14 & 4.63 & 2.07 \\
\hline 35. & L5 x T4 & -2.28 & -0.00 & - & $-6.56^{* *}$ & -0.00 & - & -1.29 & 1.37 & 1.16 & - & - & -1.99 \\
\hline 36. & L6 x T4 & -3.51 & -2.10 & - & -3.63 & -2.10 & - & $-6.56^{* *}$ & 0.58 & $-11.61 * *$ & - & - & -7.57 \\
\hline 37. & L7 x T4 & -3.39 & -3.39 & -0.84 & $-5.80^{*}$ & -3.39 & -0.84 & -3.63 & -0.71 & 6.59 & 5.24 & 2.65 & 5.98 \\
\hline 38. & L8 $\times$ T4 & $-4.78^{* * *}$ & -3.39 & -0.84 & -4.11 & -3.39 & -0.84 & $-5.80^{*}$ & -0.92 & 8.60 & 3.48 & - & 1.65 \\
\hline 39. & L9 x T4 & $-5.59 * *$ & -3.27 & -0.72 & 1.13 & -3.27 & -0.72 & -4.11 & 0.88 & 5.51 & 5.44 & 0.24 & 0.84 \\
\hline 40. & $\mathrm{~L} 10 \times \mathrm{T} 4$ & 2.97 & - & - & & - & - & 1.13 & -1.87 & $12.62 *$ & 6.77 & 1.50 & -2.19 \\
\hline
\end{tabular}


Table.3 Extent of heterosis and inbreeding depression for fruit diameter $(\mathrm{cm})$ fruit weight $(\mathrm{Kg}$.$) , fruit$ flesh thickness $(\mathrm{cm})$ and rind thickness $(\mathrm{mm})$

\begin{tabular}{|c|c|c|c|c|c|c|c|c|c|c|c|c|c|c|c|c|c|}
\hline \multirow[t]{2}{*}{ S.N. } & \multirow[t]{2}{*}{ Crosses } & \multicolumn{4}{|c|}{ Fruit diameter $(\mathbf{c m})$} & \multicolumn{4}{|c|}{ Fruit weight (Kg.) } & \multicolumn{4}{|c|}{ Fruit flesh thickness (cm) } & \multicolumn{4}{|c|}{ Rind thickness $\mathrm{mm}$} \\
\hline & & MPH & $\mathrm{HB}$ & EH & ID & MPH & $\mathrm{HB}$ & EH & ID & MPH & $\mathrm{HB}$ & EH & ID & MPH & HB & EH & ID \\
\hline 1. & L1 x T1 & -5.08 & - & - & 0.27 & -2.95 & - & - & -6.66 & 16.47 & 15.12 & - & -2.42 & -0.68 & - & - & $27.74 * *$ \\
\hline 2. & L2 $\times$ T1 & 0.59 & - & - & -13.04 & 2.26 & 0.22 & - & -6.00 & $16.36^{*}$ & 7.94 & 1.92 & -8.30 & -10.70 & - & -5.61 & 13.67 \\
\hline 3. & L3 $\times$ T1 & -5.87 & - & - & 3.12 & $-12.47 *$ & - & - & 1.99 & 1.58 & - & - & -2.89 & 18.19 & - & - & 9.80 \\
\hline 4. & L4 x T1 & 10.97 & 0.56 & - & $16.99 *$ & 1.00 & 0.09 & - & 9.58 & -2.00 & - & - & $-25.00 *$ & 2.93 & - & - & 9.76 \\
\hline 5. & L5 x T1 & -4.80 & - & - & -0.89 & 6.76 & 6.64 & - & 7.74 & 16.28 & 13.64 & - & -19.00 & -15.40 & -13.58 & - & -12.23 \\
\hline 6. & L6 $\times$ T1 & 3.07 & - & - & 7.39 & -0.80 & - & - & -3.11 & -1.10 & - & - & $-39.82 * *$ & 3.91 & - & - & 11.28 \\
\hline 7. & L7 x T1 & 4.80 & 0.84 & - & 4.17 & -11.16 & - & - & 2.70 & 8.64 & 4.76 & - & $-27.95^{*}$ & -11.20 & -4.63 & - & -13.99 \\
\hline 8. & L8 $\times$ T1 & 13.29 & 5.04 & 0.00 & -1.28 & $-12.49 *$ & - & - & $-22.19 *$ & 30.00 ** & $23.81 *$ & 0.00 & 6.73 & 15.66 & - & - & 11.76 \\
\hline 9. & L9 x T1 & -2.22 & - & - & -9.06 & -4.21 & - & - & 14.39 & 7.69 & 0.00 & - & -10.00 & -11.94 & -9.50 & - & 2.92 \\
\hline 11. & L1 x T2 & -3.75 & - & - & -5.85 & 1.10 & - & - & -7.85 & -9.13 & - & - & -3.11 & 8.71 & - & - & -2.10 \\
\hline 12. & L2 $\times$ T2 & $14.58^{*}$ & 10.82 & 0.27 & 1.33 & -0.44 & - & - & 6.40 & 7.24 & 7.13 & 1.15 & 8.17 & 5.54 & - & -4.55 & 13.83 \\
\hline 13. & L3 $\times$ T2 & $33.98 * *$ & $31.15^{* *}$ & $18.67 * *$ & 7.87 & 7.45 & 5.62 & 0.29 & 9.93 & 11.92 & 9.18 & 2.88 & -1.12 & 8.82 & - & -0.18 & -1.85 \\
\hline 14. & $\mathrm{~L} 4 \times \mathrm{T} 2$ & 13.78 & 5.51 & - & -2.51 & $13.20 *$ & 11.95 & 6.30 & $15.61 *$ & -9.09 & - & - & $-28.18^{*}$ & -1.80 & - & - & -9.42 \\
\hline 15. & L5 $\times$ T2 & 5.75 & 3.99 & - & 0.41 & -3.12 & - & - & 2.26 & 5.38 & 0.00 & - & -10.31 & -12.08 & - & - & -9.03 \\
\hline 16. & L6 $\times$ T2 & 2.81 & 0.80 & - & 8.77 & -2.82 & - & - & 9.30 & -8.16 & - & - & -15.33 & -3.28 & - & - & -22.15 \\
\hline 17. & L7 x T2 & 6.68 & 5.22 & - & 7.42 & $-18.21^{* *}$ & - & - & -4.02 & 11.36 & 0.00 & - & $20.00 *$ & -2.65 & - & - & -4.63 \\
\hline 18. & L8 $\times$ T2 & $26.03 * *$ & $19.66 *$ & 8.27 & 0.74 & 2.35 & - & - & 1.07 & 14.71 & 1.84 & - & -0.60 & -9.36 & -3.45 & -6.68 & 14.31 \\
\hline 19. & L9 $\times$ T2 & 12.85 & 9.64 & - & 5.38 & $-21.96 * *$ & - & - & 8.86 & 4.08 & 4.08 & - & -17.06 & -6.25 & - & - & -7.13 \\
\hline 20. & L10 x T2 & 8.33 & 6.69 & - & -3.87 & -0.23 & - & - & -10.26 & -8.19 & - & - & -6.37 & 2.55 & - & - & 8.72 \\
\hline 23. & L3 $\times$ T3 & 13.62 & 11.69 & - & -2.48 & -1.14 & - & - & 5.26 & 1.55 & - & - & $-20.87^{*}$ & 6.24 & - & - & 15.02 \\
\hline 24. & L4 $x$ T3 & 14.60 & 10.22 & - & -8.35 & -11.82 & - & - & 2.99 & -1.96 & - & - & -11.11 & -7.79 & -6.57 & - & 5.50 \\
\hline 25. & L5 x T3 & -12.18 & - & - & -0.68 & 5.46 & 2.54 & - & 11.26 & 0.00 & 0.00 & - & -3.18 & -3.78 & -0.56 & - & 18.46 \\
\hline 26. & L6 x T3 & 5.31 & 3.37 & - & 1.48 & -8.81 & - & - & 3.20 & 5.38 & 0.00 & - & -7.96 & 3.78 & - & - & $24.76^{*}$ \\
\hline 27. & L7 x T3 & $17.08^{*}$ & 14.24 & 0.53 & 3.50 & -9.65 & - & - & 2.74 & 10.12 & 3.86 & - & -0.44 & -4.26 & - & - & 10.28 \\
\hline 28. & L8 $\times$ T3 & $20.84 * *$ & 19.11* & - & 6.42 & 2.45 & 0.76 & - & 3.33 & 14.63 & 6.82 & - & -18.94 & 3.43 & - & - & $26.01 *$ \\
\hline 29. & L9 $\times$ T3 & $18.30 *$ & $17.19 *$ & 0.00 & -7.20 & 0.94 & - & - & -14.48 & -3.23 & - & - & -19.11 & -14.08 & -12.72 & - & 3.20 \\
\hline 30. & L10 $\times$ T3 & 7.62 & 5.17 & - & -9.83 & 6.16 & 5.78 & - & 5.23 & 6.56 & - & 0.00 & -5.96 & $-18.10 *$ & -16.91 & - & $-26.22 *$ \\
\hline 31. & L1 x T4 & 8.91 & 5.28 & 1.07 & 2.90 & -0.65 & - & - & 5.88 & -5.62 & - & - & -21.43 & -5.03 & -4.79 & - & 11.40 \\
\hline 32. & L2 $\times$ T4 & 6.94 & 0.56 & - & -0.83 & -0.84 & - & - & 1.79 & -7.47 & - & - & -13.64 & 10.77 & - & - & -3.09 \\
\hline 33. & L3 $\times$ T4 & 9.78 & 4.44 & 0.27 & 1.06 & 6.25 & 5.08 & - & 3.88 & 14.47 & 13.73 & 1.92 & -16.04 & -2.30 & - & - & -10.10 \\
\hline 34. & L4 $\times$ T4 & 11.38 & 0.56 & - & -0.28 & 2.28 & 0.54 & - & 4.19 & -3.20 & - & - & $-26.87^{*}$ & 0.60 & - & - & 15.11 \\
\hline 35. & L5 $\times$ T4 & 6.61 & 5.28 & 1.07 & 9.02 & -6.05 & - & - & $20.79 *$ & 0.00 & - & - & -8.00 & -8.21 & -4.09 & - & -5.90 \\
\hline 36. & L6 x T4 & $-15.60 *$ & - & - & -0.86 & -10.06 & - & - & 5.32 & 1.05 & - & - & -10.00 & -2.79 & -1.05 & - & -7.89 \\
\hline 37. & L7 x T4 & 0.58 & - & - & -7.78 & 3.34 & - & - & -7.69 & 5.88 & - & - & -15.56 & 6.45 & - & - & $20.95 *$ \\
\hline 38. & L8 $\times$ T4 & 6.47 & - & - & -6.78 & -5.41 & - & - & 1.00 & $21.43 *$ & 10.87 & - & -3.33 & -8.33 & -3.11 & - & -14.11 \\
\hline 39. & L9 $\times$ T4 & 10.59 & 4.44 & 0.27 & -2.66 & -4.13 & - & - & $18.61^{*}$ & -3.16 & - & - & -9.78 & -16.42 & -15.99 & - & -3.90 \\
\hline 40. & L10 x T4 & $18.72 * *$ & 13.61 & 9.07 & 9.05 & -4.70 & - & - & -2.30 & -1.61 & - & - & -5.51 & -7.13 & -6.78 & - & 5.53 \\
\hline
\end{tabular}


Similarly, heterosis over better parent is negative but not significant for any of the crosses. The extent of inbreeding depression is also negative and significant over the economic parent but data of inbreeding depression over mid parent is displayed in same table where inbreeding depression ranged from -0.27 in cross KBGL-21 X Pusa Samaridhi to highest range (9.33) of inbreeding depression found in cross Kalyanpur Long Green x Narendra Rashmi followed by Narendra Pratibha $X$ Pusa Samaridhi (-7.54). For days to $50 \%$ male flower, it is obvious that the heterosis over mid parent was significant in only one crossAzad Harit x Narendra Rashmi where significant inbreeding depression was also observed. The maximum inbreeding depression (-9.89) was noticed in the cross KBGL-22 x Kashi Ganga followed by (8.99) in cross Narendra Madhuri X Arka Bahar (Table 2). The extent of heterosis was negative and significant in 10 crosses over mid parent whereas heterosis over better parent was observed from $-4.07 \%$ to $5.33 \%$. Economic heterosis is not observed for this character. The present observations are in agreement with the findings of Singh (2008). Inbreeding depression for these two characters over the mid parent range from $8.18 \%$ in crosse KBGL-19 x Kashi Ganga followed by KBGL-21 x Arka Bahar. Heterosis for days to first fruit harvest over better parents ranged from $-6.98 \%$ in cross KBGL-21 x Narendra Rashmi. But inbreeding depression was only $-1.31 \%$. This indicates that predominance of heterosis in a cross may not be necessarily associated with high degree of inbreeding depression due to major role of additive gene action controlling a particular character. This is also true for $2^{\text {nd }}$ cross KBGL-21 x Narendra Rashmi. For fruit length the positive and significant heterosis over the mid parent was shown by two crosses i.e. Purnima S-1 X Pusa Samaridhi and Purnima S-1 x Arka Bahar, whereas negative heterosis was shown again by two crosses i.e. Purnima S-1 X Pusa Samaridhi and Narendra Madhuri x Pusa Samaridhi. These crosses also showed high degree of inbreeding depression. The low magnitude of inbreeding depression indicates that there is greater contribution of additive gene action in expression of heterosis in the cross like Nrarendra Madhuri x Pusa Samaridhi. In some cases low heterosis is linked with high degree of inbreeding depression like in cross Narendra Pratibha $\mathrm{x}$ Arka Bahar and in cross KBGL-21 x Narendra Rashmi. This suggests that there is pre ponderance of additive gene action operating in these crosses. Positive and significant heterosis has been displayed by 8 crosses over mid parents. Of these, one cross Narendra Pratibha $\mathrm{x}$ Narendra Rashmi has also shown maximum inbreeding depression, where magnitude of heterosis is also highest.

For fruit weight, four crosses showed positive and significant heterosis where the magnitude of inbreeding depression was also high (Table 3). For fruit flesh thickness, significant heterosis over mid parent as well as better parenthas been noted. However, two more crosses also showed positive heterosis over mid parent with greater expression of heterosis. In general, high heterosis is not linked with high inbreeding depression in this case, suggesting thereby that there is greater role of additive gene action in the expression of heterosis. The presence of significant heterosis for yield and yield components of bottle gourd have also been reported earlier by Singh et al., 1998 and Kumar et al., 1999. The expression of heterosis was most evident in the present study for vine length, number of primary branches, fruit length, number of pistillate flowers, number of fruits per plant, and yield per plant as compared to other characters studied. The results of also revealed that the crosses IC-92362 x Pusa Naveen and IC-92462 x Pusa Naveen exhibited high heterosis for yield and also 
other yield components viz., fruit diameter, vine length and number of branches/plant indicating the additive or synergistic effect of the components characters on fruit yield (Pal et al., 2005)

For rind thickness, most of the crosses showed negative heterosis which is desirable for bottle gourd. For vine length, heterosis has been noted over mid and better parent in three cosses (Nrarendra Madhuri x Pusa Samaridhi, Azad Harit X Pusa Samaridhi and KBGL-1 X Kashi Ganga) and there was positive inbreeding depression for this character in these crosses, suggesting that there was greater role of dominance or epistatic gene action in controlling the expression of this trait.

\section{References}

Allard, R.W. 1960. Principles of plant Breeding. John Wiley and Sons. Inc., U.S.A. p. 485.

Hayes HK, Immer IR, Smith DC. Method of Plant Breeding. McGraw Hill Company Inc., New York, 1955, 535.

Janakiram, T. and Sirohi, P. S., 1989. Heterosis studies in round fruited bottle gourd. Madras Agricultural Journal, 76 (6): 339-342.

Janaranjani, K. G., Kanthaswamy, V. and Kumar, R. S., 2016. Heterosis,
Combining Ability and Character Association in Bottle Gourd for Yield Attributes. International Journal of Vegetable Science., 22(5) 490-515.

Kempthorne, O. 1956.The theory of the diallel cross. Genetics. 41: 451-59.

Pal, S. N., Ram, D.., Pal, A.K.., Rai, M., (2005) Heterosis studies in bottle gourd (Lagenaria siceraria (Molina) Standl.). Indian Journal of Horticulture 3 (62) pp-253-256.

Shull, G.H. 1914. The composition yield of maize. Rpt. Amer. Breeder's Assoc. 4: 296-301.

Singh, K.P., Choudhury, D.N., Mandal, G. and Saha, B.C. (2008). Genetic variability in bottle gourd (Lagenaria siceraria (Molina) Standl.). Journal of Interacademicia, 12: 159-163

Sinha, S., Mishra, S. B., Paramhans, P., Pandey S. S., 2018 Heterosis and inbreeding depression for yield and yield component in interaspecific crosses of Vigna, International Jounral Current Microbiology Applied Science. Special Issue-7: 4912-4917,

Thakur, P., Singh, J., Nair, S.K. and Priyadarsini S.D., 2017. Correlation and path analysis in bottle gourd [Lagenaria siceraria (Mol.) Standl.]. International Journal of Current Microbiology and Applied Sciences., 6 (12): 1478-1485,

\section{How to cite this article:}

Sandeep K. Mauriya and Pal, A. K. 2021. Line x Tester Analysis for Heterosis and Inbreeding Depression in Bottle Gourd (Lagenaria siceraria (Mol) Standl.). Int.J.Curr.Microbiol.App.Sci. 10(02): 631-637. doi: https://doi.org/10.20546/ijcmas.2021.1002.075 